\title{
Patient-Centred Coordinated Care in Times of Emerging Diseases and Epidemics
}

\section{Contribution of the IMIA Working Group on Patient Safety}

\author{
E. Borycki' ', E. Cummings² J. W. Dexheimer ${ }^{3}$, Y. Gong ${ }^{4}$, S. Kennebeck ${ }^{5}$, A. Kushniruk', \\ C. Kuziemsky ${ }^{6}$, K. Saranto ${ }^{7}$, J. Weber ${ }^{1}$, H. Takeda ${ }^{8}$ \\ I School of Health Information Science, University of Victoria, Victoria, British Columbia, Canada \\ 2 School of Health Sciences, Faculty of Health, University of Tasmania, Hobart, Tasmania, Australia \\ 3 Division of Emergency Medicine, Division of Biomedical Informatics, Cincinnati Children's Hospital \\ Medical Center, Cincinnati, OH, USA \\ 4 School of Biomedical Informatics, University of Texas Health Science Center at Houston, Houston, Texas, USA \\ ${ }^{5}$ Division of Emergency Medicine, Cincinnati Children's Hospital Medical Center, Cincinnati, OH, USA \\ 6 Telfer School of Management, University of Ottawa, Ottawa, Ontario, Canada \\ 7 Department of Health and Social Management, University of Eastern Finland, Kuopio, Kuopio, Finland \\ ${ }^{8}$ Graduate School of Health Care Sciences, Jikei Institute, Osaka, Japan
}

\begin{abstract}
Summary
Objectives: In this paper the researchers describe how existing health information technologies (HIT) can be repurposed and new technologies can be innovated to provide patient-centered care to individuals affected by new and emerging diseases. Methods: The researchers conducted a focused review of the published literature describing how HIT can be used to support safe, patient-centred, coordinated care to patients who are affected by Ebola (an emerging disease).

Results: New and emerging diseases present opportunities for repurposing existing technologies and for stimulating the development of new HIT innovation. Innovative technologies may be developed such as new software used for tracking patients during new or emerging disease outbreaks or by repurposing and extending existing technologies so they can be used to support patients, families and health professionals who may have been exposed to a disease. The paper describes the development of new technologies and the repurposing and extension of existing ones (such as electronic health records) using the most recent outbreak of Ebola as an example.
\end{abstract}

\section{Keywords}

Patient safety, health information technology, Ebola virus, electronic health records, technological innovations

Yearb Med Inform 2015;10:207-15

http://dx.doi.org/10.15265/YY-2015-019

Published online June 30, 2015
Patient-Centered, Coordinated Care has been associated with improved patient outcomes [1] reductions in medical errors [2] and improved patient and family satisfaction with the quality of the care provided by healthcare organizations [3-5]. Gulliford and colleagues [6] define patient-centred care as "providing care that is respectful of and responsive to individual patient preferences, needs, and values, and ensuring that patient values guide all clinical decisions." When patient care is coordinated, healthcare teams can work more effectively together, supporting patient and family decision-making in all settings where the patient is cared for (e.g. physician office, home, hospital and long-term care). This in turn improves communication and coordination between health professionals, patients and their families, leading to improved quality of care. Health information technology (HIT) has been identified by the IOM (Institute Of Medicine) as a tool that can support communication and coordination of care, thereby improving patient-centred care [7].

Historically, HIT has been used passively to support patient-centered care in hospital settings by facilitating real-time access to laboratory and diagnostic imaging results, clinical documentation and decision support tools specific to a patients' acute and/or chronic illness events. HIT has been developed to support patient-centered, evidence based care in patients with previously diagnosed acute and chronic illnesses. Over time clinical research has advanced the development of specific patient-centered interventions for those people who are affected by acute and chronic illnesses [8]. In addition, research has led to the development of electronic tools that enable patient-centered care [7, 11].

Much of this HIT research has focused on electronic tool development for known acute and chronic illnesses [7]. For HIT to fully support patient-centred approaches to acute and chronic disease management there is a need for health and biomedical informatics researchers to develop and improve upon current HIT through continuous development, improvement, iteration, testing, optimization and evaluation of technology using measures of patient centeredness [10]. Such work involves investment by all those who benefit from patient-centred HIT - governments, vendors, healthcare organizations, health professionals, patients and their families [7].

Research and use of HIT in the context of dealing with known acute and chronic illnesses has advanced significantly over the past decade. However, there has been less interest and research in understanding how HIT can support patient-centred care when patients and their families are affected by new/ emergent diseases such as Ebola. In this paper 
we discuss how HIT can be repurposed and developed to provide patient-centered support to individuals who are affected by new/ emerging diseases or diseases about which we have limited knowledge or experience. We also discuss the future of technologies that will support patient-centred care in these contexts. Efforts around the Ebola virus will be used as an example. We will also consider some of the issues and challenges associated with addressing such diseases from a HIT perspective and how our learning's can be used to address emerging diseases and diseases that may require additional research to understand and treat into the future.

\section{Background}

Patient-centred HIT in hospital settings is more researched, easily implemented and evaluated in comparison to the introduction of those HIT currently used to support treatment and management of patient-centred approaches towards the management of new/ emerging diseases [9]. Emerging diseases are not typically encountered by technology designers and HIT professionals. New and emergent diseases that we encounter today in our world of modern epidemics include Severe Acute Respiratory Syndrome (SARS), the Avian flu and Ebola [12]. The development of HIT that supports patient-centred care for those patients and families affected by new/emerging diseases, especially in regions of the world where HIT and health professionals have limited knowledge or experience with new/emerging diseases, is critical to developing and exchanging knowledge about these diseases [13]. Such technologies are also needed to support patients who are diagnosed with acute and chronic illnesses. Given the new and emergent nature of some diseases one might conclude that HIT used to support health professionals in the management of these diseases is less patient-centred and therefore less effective in supporting patient-centred care. Yet, HIT can evolve and be used to support the development of patient-centred care. For example, in the early days of the global outbreak of SARS, there was little known about the disease. Yet, health and biomedical informatics researchers developed electronic tools and repurposed existing technologies such as electronic health records (EHRs) to effectively support clinicians and biomedical researchers. Here, HIT researchers developed virtual environments where healthcare professionals could communicate, collaborate, and document information about the treatment and management of SARS [14]. This included patient-centred approaches to the management of the disease. Clinicians, patients and their families were involved in decision making even as new information about the prevention and management of this disease was emerging using electronic tools. This could only be done through HIT such as the Internet, EHRs, decision support systems, and virtual collaborative environments [15]. Today, the Ebola epidemic has presented the health and biomedical informatics community with similar challenges - to develop HIT that allow for exchange and communication across care settings which still allows for patient-centred care. In the next section of this paper we provide a brief background about Ebola.

\section{Ebola}

Ebola is a rare and fatal illness. Early recognition and diagnosis of the disease in individuals, who have been exposed to the virus, is important from a public health, patient, and family perspective. Ebola's incubation period is from 2 to 21 days from the time of infection to the onset of symptoms. The symptoms of the disease include an "elevated body temperature or subjective fever or symptoms, including severe headache, fatigue, and muscle pain" [16], followed by vomiting, diarrhea, rash, symptoms of kidney impairment and liver function and (in some cases) both internal and external bleeding (e.g. oozing gums, bloody stools). Laboratory findings include "low white blood cell and platelet counts and elevated liver enzymes" [17].

The Ebola virus first appeared in 1976 in Nzara (Sudan) and in Yambuku (Democratic Republic of Congo). The virus takes its name from river Ebola which is near the village where the disease first appeared. The current outbreak, which began in March of 2014 , is the largest. There have been more cases and deaths from Ebola in the current outbreak than all other outbreaks combined. The disease has also spread from Guinea to Sierra Leone, Liberia, Nigeria, Senegal, the United States of America, Spain and Mali [17] and "Germany, Norway, France, Italy, Switzerland and the UK have all treated patients who contracted the virus in West Africa" [18]. To date, 6,856 people have died (including suspected, probable and confirmed cases of the disease) [18].

Fruit bats from the Pteropodidae family are thought to be the hosts for the virus, and the virus is transmitted to humans from bats through "close contact with the blood, secretions, organs and other bodily fluids of infected animals such as fruit bats, monkeys, forest antelope and porcupines found ill or dead in the rainforest". Transmission of Ebola between humans occurs via direct contact with "(broken skin or mucous membranes) with the blood, secretions, organ or other bodily fluids of infected people, and with surfaces and materials (e.g. bedding and clothing) contaminated with these fluids" [18].

Healthcare workers can become infected with Ebola when they are in close contact with those affected by the disease and infection control precautions are not fully employed [17]. Infection control precautions include wearing suits, goggles, gloves, boots and protective head gear. There is no U. S. Food and Drug Administration approved medication or vaccine that can be used to treat the disease. Current approaches to the treatment of Ebola include the management of symptoms and complications arising from the disease. For those who have contracted the disease, survival is dependent on good supportive care and the individual's own immune system response to the disease. Supportive care includes: (a) "providing intravenous fluids (IV) and balancing electrolytes (body salts), (b) maintaining oxygen status and blood pressure, and (c) treating other infections if they occur" [16]. Vaccines and treatments for Ebola are currently under development, but none have been fully tested for their safety and/or their efficacy [16]. People, who recover from Ebola, develop antibodies that may last at least 10 years. "It is not known 
if people who recover are immune for life or if they can become infected with a different species of Ebola. Some people who have recovered from Ebola have developed longterm complications, such as joint and vision problems" [16]. Prevention of the spread of Ebola in the general population involves education about the disease and its spread, identifying those who have had contact with infected individuals and early identification/ isolation of those who develop symptoms (including providing supportive care) [16]

HIT has played a significant and important role in the treatment of Ebola. Some HIT has been modified to support health professionals' use of the technology in providing patient-centred care to patients who are affected by the disease. This work by HIT professionals represents an example of how technologies can be modified to support public, patient and family health in response to a significant outbreak of a disease. It also takes into account hardware and software, clinical content, people (including human interface, communication), organizational (such as workflow) and contextual issues (including external rules, regulations and pressures) [19]. In the next section of this paper we describe how HIT has been innovated, modified and optimized to support care of patients, their families and the public in a patient centric approach.

\section{Health Information on the Web (Government and WHO Websites)}

The World Wide Web (WWW) has become a pivotal public resource for attaining and sharing health-related information. While information-seeking habits differ, depending on the nature of the health concern as well as other factors, such as demographics and health literacy, the majority of health consumers trust Internet search engines (e.g., Google) with retrieving an initial set of relevant information sources [20-22]. Public interest about emerging diseases often coincides with related news-making events such as the SARS, the Avian Flu and Ebola crisis in Africa. Google trends analysis reports two major spikes of searches related to Ebola (see Figure 1): The first spike in August 2014 coincides with the WHO declaring Ebola an international public health emergency, while the second, larger spike follows news about the first cases of Ebola discovered in the U.S. (points $F$ and C). Yet, many patients, families and health professionals often use the Internet as a starting point for gathering information about a disease that may affect them or the health of their families. For example, researchers have identified that the Internet is a source of health information for $87 \%$ of Americans [23].

Using general-purpose Internet search engines for retrieving health information by patients and families has the drawback of delivering search results of unknown quality. Moreover, the sheer number of returned resources and the determination of their relevancy to the information seeker's query objectives may pose a challenge. For example, a medical practitioner seeking practice guidance on an emerging disease may be exposed to a large number of irrelevant, news-related search results. Purpose-dedicated search engines for retrieving trusted health information provide an alternative, e.g., services provided by the Health on the Net Foundation (www. healthonnet.org) [24]. They provide certified information content that is targeted towards particular audiences, including healthcare consumers and medical professionals. However, their popular adoption remains low and the majority of information seekers use

other strategies to filter credible and relevant results from general Internet search engines, e.g., a critical evaluation of the organization providing the information.

Government Web sites such as the U.S. Centers for Disease Control and Prevention (CDC) and international healthcare organizations like the World Health Organization (WHO) are considered among the most trusted sources of information [25]. However, the content presented on these sites at the time of the initial outbreak had limited value for patients. Archived versions of the WHO web site indicate that a prominent patient-centred focus has only been adopted seven months after the initial outbreak (retrieved though $\mathrm{http} / /$ archive.org/web/). Before this time, patient information content was mixed with WHO news releases, guidelines for professionals, WHO partner organization, researchers and other audiences. The current WHO Web site has a clearly demarcated patient-centred section (along with two other sections, centered on providers and partner organizations, respectively). It leverages Web-based patient-centred communication tools such as Twitter and Facebook to propagate important health facts about the disease while it emerges. More localized information is posted on individual governmental Web sites. For example, the CDC provides focussed information content for parents, teachers, travelers, etc. The disease has also spawned the creation of new patient-centred Web communities that collect and make

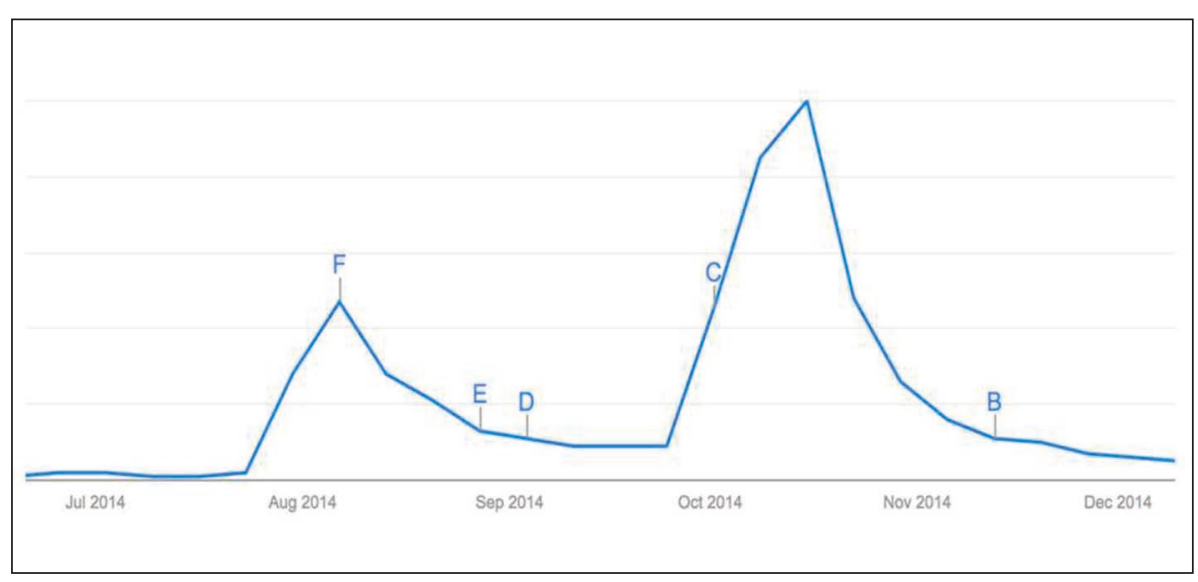

Fig. 1 Public Web searches for Ebola (Google) 
available disease-related information for the public, e.g., whebola.com, ebolafiles. com, ebolabreakoutnews.com. Notably, the emergence and popularity of many of these communities has bypassed the traditional Web (and Web search engines) and hinged on social networks instead. Whebola.com, for example, is not easily retrievable through classical Web search engines (like Google) but very prominent with searches on for \#ebola on Twitter. This indicates the increasing importance of focussing on social networking technologies in patient-centric health information delivery. It also indicates that the growing reliance on social networks as a way to retrieve and communicate information has created new risks with respect to potential misinformation and patient safety. Innovative approaches to ensuring trustworthiness are required for navigating the social health Web.

\section{Mobile Applications}

Today, the use of mobile applications in conjunction with smartphones is significant. Research suggests that $75 \%$ of individuals use a mobile or smart phone [26], with 2.8 million using that technology in conjunction with a mobile application to self-manage their health and disease [27]. There has also been an emergence of software applications that aid patients' and families in their health related decision-making (as in cases of when to seek medical attention). There has been a proliferation of mobile software applications in these areas, especially those that focus on self-management of diet, exercise, diabetes, hypertension etc. Some mobile healthcare application designers have recently begun to focus on tracking and information about the signs and symptoms of Ebola.

To date, there has been limited Ebola specific symptom monitoring apps developed. However, as the incubation period for Ebola is typically up to 21 days and the early symptoms of the disease are symptoms that are common to many diseases. Mobile applications have been developed for both Android and IOS platforms, with the majority of them being information sharing applications or providing outbreak tracking maps for Ebola. Other mobile applications typically used for social media purposes are also another way in which Ebola related information can be accessed and reviewed (as outlined in the previous section of this paper). Social media mobile applications have been used to push information about Ebola via Twitter ${ }^{\circledR}$, Youtube ${ }^{\circledR}$, Facebook ${ }^{\circledR}$ etc.

It is interesting and worthy to note that whilst Africa is one of the fastest growing mobile phone markets it has the lowest rate of smartphone technology penetration in the world. In January 2014 it was estimated that $12 \%$ of the African population had smartphones, with the majority of smartphone owners living in the wealthier, urban areas [28]. Evidently this low penetration provides difficulties in the use of mobile applications for symptom monitoring in the Ebola stricken areas of Africa versus other countries which have higher rates of mobile application usage.

A number of smartphone manufacturers have donated smartphones to Ebola affected countries. These smartphones are primarily for the use of healthcare workers and are used by them to collect data that will assist in monitoring and planning in the epidemic. Further important uses of mobile phone technology are their use for connecting the sick with their relatives. This is undertaken using basic mobile phones so that they can talk with their families. This contact is particularly important for patients in isolation. Mobile phones are also being used to share and receive information via short message service (SMS). Whilst most systems are designed to share information with some systems, many mobile applications have the ability to receive data that can be used for tracking outbreaks of Ebola [28, 29, 43].

\section{Electronic Health Records}

After the initial Ebola case in the United States in Dallas, Texas, public attention became focused on the EHR. The EHR was blamed and believed to be a contributing factor in the initial missed diagnosis [30]. In truth, the EHR itself was not to blame, but the answer was likely more in the way the EHR collected and presented critical data to the medical team and the current state of organizational policies where the technology was concerned. The EHR should have helped to better identify a patient for whom a clinical concern should be raised, particularly when a disease is rare and not on the mind of providers (as was the case for the medical team in Dallas at the time). The concern was not that the critical data had not been collected, but that it had been placed in a relatively unobtrusive area that was easy to skip when the EHR was reviewed. This in turn led to confusion where the patient's international travel was recorded and whether the relevant information was effectively communicated to all members of the healthcare team (http://res.dallasnews.com/ interactives/duncan-timeline/). This would constitute an example of what Borycki and colleagues term a "technology-induced" error [31], which is an error that results from human factors issues and deficiencies whereby a health information system may lead a health professional to make an error (which could be an error of omission, such as omitting to read key information, or commission). Such errors do not result from errors in programming but rather emerge in the complex interaction of health information systems with end users in the busy and often hurried context of clinical practice. Indeed a key aspect of both EHRs systems and public health systems is their level of usability, with systems and user interfaces that are difficult to navigate or use leading to a higher probability of technology-induced error [32]. In response to the problem in this example, the hospital then reported updating their EHR to collect additional screening information in a more rigorous fashion to help identify high-risk patients [33].

\section{Electronic Decision Support Systems}

Electronic decision support systems (DSS) can be used to aid providers in identifying these rare patient events by streamlining data collection, and then organizing and displaying data to the appropriate personnel. 
DSSs are part of every modern EHR implementation and are defined as "any program designed to help health-care professionals make clinical decisions;" [34]. DSS support can cover many aspects of care including patient or disease-specific recommendations, [35] information management, [36] and evidence based guideline compliance [37]. Given the vast amount of data collected during a patient encounter, leveraging the EHR to provide directed, timely, and valuable information is an important goal.

More than $70 \%$ of US hospitals have EHRs, [38] and most of these contain a degree of decision support. In a recent article in Journal of the American Medical Informatics Association, the concept of applications either integrated within or tightly combined with the EHR to deliver decision support is proposed as a way to improve healthcare quality [39] The applications can be small modules for disease-specific screening, or larger scale interventions designed to implement standardization across a healthcare system. While patient or disease specific support is difficult to implement initially in an integrated manner, this method of providing decision support has the benefit of being relatively easy to edit and can be amended with new science or known screening questions once integrated, therefore providing a quick implementation strategy for the identification of emerging diseases.

Traditional DSS relies on alerts that are in-line with the standard EHR templates and creates pop-ups to alert providers that disrupt the workflow. When coded information is collected in the EHR about travel or other designated related screening information, an active pop-up alert for patients such as the one illustrated in figure 2, can be displayed to the relevant provider to acknowledge the risks and initiate any desired behaviours or treatment pathway. As passive decision support, additional reminders can be added to the patient's chart in the form of informational banners or highlights. This system would work with the existing workflow of providers but interrupt (through the active alert) only when patients screen positive. It also has the drawback that it needs to be adjusted. In this specific case, when patients traveled to one of four specific countries, the alert fires. If, as is prevalent in infectious disease, the

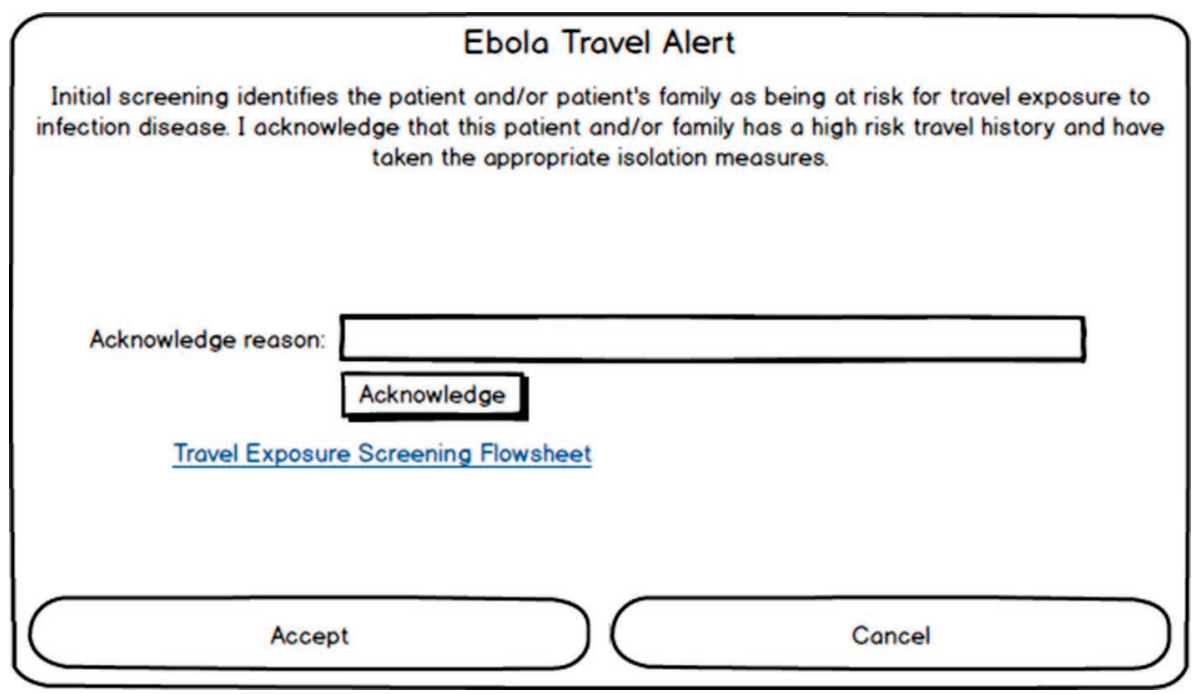

Fig. 2 A sample pop-up alert for patients pre-screened for travel exposure and disease risk for Ebola

countries or risk factors change, the coding behind the alert must change as well. This requires more programming support. Also-if the list of risk factors grows very large (in our example, if the list of affected Ebola countries were to swell to 10-20 countries) the electronic screening would likely be much less accurate. Here, one could look towards international organizations such as the World Health Organization to maintain and update DSS content about Ebola using a service oriented architecture approach [40].

Even with these existing drawbacks, these types of alerts do provide workflow integrated reminders to providers at the point of care instead of just a passive dissemination of information. If the initial nurse for Ebola patient zero had gotten an alert on her screen when she documented western African travel, we can all assume the initial emergency department visit would have looked much different. Decision support can be one tool to help busy providers remember to screen for rare events, and give direct messaging when such a screening is positive. Alternatively, providers should also be made aware of the potential for false alarms or false positives. Here, clinicians must rely on further assessment and their clinical judgement to ascertain the presence or absence of a disease. The future of this type of support lies in leveraging systems that work with devices that patients already use, for example, smart phones. If some screening questions could be answered by text on a patient's own device and integrated into the EHR possibly even before presentation, this would increase the power and flexibility or this type of decision support.

\section{Challenges and Issues}

Although existing technologies such as the WWW, social media, EHRs and DSS can be used to support patient centric care. There are a number of challenges associated with their use. In the next section of this paper we outline issues.

\section{Electronic Health Records, Interoperability, Data Integration and Surveillance}

A key challenge in providing patient centered coordinated care delivery is coordinating care across providers and settings using a patient-centred perspective. EHRs and other health information technologies offer great potential to serve as the coordinating agent across providers and settings [41]. However, a key challenge to using EHRs for 
supporting coordinated patient centered care is operationalizing the actual coordination that needs to occur [42]. While EHRs and other HIT may adequately support defined episodes of care, they are less helpful in supporting exceptions or unintended situations such as disease outbreaks [44]. While patients may present at the emergency room during a crisis, they do not originate there, but rather they come from a clinic or home in the community.

EHRs are being used to identify outbreaks of disease. Some EHRs can be used to generate physician practice, organizational and system level statistics on the presence of symptoms of certain communicable and chronic diseases. In the case of an outbreak of disease, EHRs could be searched for information about individuals and their contacts. As in the case of identifying all the health professionals and family members that were in contact with an individual with Ebola [45]. There has been some work in developing computer algorithms to identify cases of disease based on laboratory results and information found in unstructured texts. For example, the Electronic Medical Record Support for Public Health (ESPnet) employs algorithms that use patient laboratory test results, vital sign information, prescription information and physician diagnostic coding information to identify outbreaks of disease that are public health concerns $[47,48]$. This work as also been extended to the use of algorithms that search narrative text within EHRs [49]. Such work would be important in identifying when a patient who has symptoms of Ebola is treated or admitted to a hospital. There would be a need to invest in research that would lead to the development and validation of such algorithms [45].

Currently, there exists a lack of integration between EHRs, EMRs and public health information systems which slow public health response to disease outbreaks [45]. As a consequence, there is a disconnect: we are able to adequately manage disease outbreaks in hospital, but we are not fully able to conduct ongoing disease surveillance using public health systems, thereby allowing some diseases to continually re-emerge time and time again $[47,48]$. The lack of interoperability between systems may lead to missing data that might be useful in outbreak detection [45]. While data integration between EHRs, EMRs and public health information systems is needed to track patients across multiple settings, it is more than just clinical data that needs to be integrated. Studies have described how psychosocial, behavioral, and environmental data are often of interest to public health [44]. There is a need for interoperability between these HIT for surveillance, information exchange to enhance surveillance and tracking of those that may be affected by the disease, their families and community contacts [45] Once this is achieved algorithms might be used to detect potential cases of communicable diseases [45].

Research is also needed to extend our development of search engines and data mining approaches [45] where the EHR and communicable diseases such as Ebola are concerned. In an extension of this work research had demonstrated that we can create search engines that search for the emergence of specific patterns of information and can be used to search aspects of the EHR such as the free text and narrative components of the technology [45-49].

\section{Electronic Health Record Design}

However, a more relevant issue is that HIT does not provide adequate support for management of emerging diseases. A data field such as whether a patient has travelled abroad may not be significant in usual scenarios but in the case of a disease outbreak it becomes far more significant. Clinicians often work in complex contextual conditions. Regardless of whether the Texas Ebola patient communicated information about his travels in Liberia, West Africa, in a stressful emergency room setting it can be difficult for a clinician to properly process all the requisite information. HIT needs to be designed with sufficient flexibility to enable alerts and reminders to be quickly configured to raise attention to relevant data fields and to ensure the fields are communicated appropriately. To design HIT to support monitoring and management of disease outbreaks will require a rethinking in HIT. More specifically, we need to better incorporate context in HIT design (i.e. physician office, hospital etc.). Contextual rethinking needs to be done.

\section{Human Factors Issues}

Human factors have been identified as a key aspect of effective design and use of not only general types of health information systems and technologies but also emerging systems such as public health surveillance systems [9]. Such systems are being developed to integrate information from multiple sources including information about prior disease cases with EHR data and new incoming data about specific client cases and epidemic spread. The SARS epidemic fuelled the creation and deployment of such systems in Canada and internationally. However, the usability and effectiveness of such systems will need to be considered and optimized for them to achieve their promise. This includes consideration of providing the most effective user interfaces for entering new data (e.g. from points of entry in a country, offices, and clinical settings across regions or countries), retrieving patient and case data, and also for providing effective and timely alerting about potential epidemics, disease spread and counter measures. To address these challenges, methods from usability engineering [50] are beginning to be applied throughout the systems development life cycle of health surveillance systems to ensure their design and deployment are effective and such systems lead to adoption and proper use when epidemics and public health emergencies arise. This has included usability inspection of the user interfaces of such systems, usability testing involving observing representative users of such systems (e.g. public health officers, clinicians, managers etc.) and clinical simulations, where systems are tested under simulated conditions and environments (e.g. simulations of public health emergencies) [31]. In addition, software and applications targeted for use by the public should be optimized by applying usability engineering principles and methods to ensure information can be retrieved and user interfaces are both usable and understandable. 


\section{Policy and Organizational Issues}

Policy and organizational issues are of particular concern during the early days and weeks of an outbreak especially when health professionals are only beginning to learn about the signs and symptoms of a new disease, the mode of its transmission and the best ways to treat and manage the disease. Existing information, policies, and procedures (and the technologies that enact them such as public health information systems, telehealth systems and EHRs) may not fully account for all information about the emerging disease. Information about a new or emerging disease may also change on an hourly or even a daily basis as new information emerges about the treatment of the disease and is communicated among country, state and provincial governments. In some cases international and national agencies may provide collaborative tools that allow for health professionals to exchange information and experiences with these emerging diseases to enhance development of protocols (as in the case of SARS where international experts around the world worked together on understanding how to best identify and treat the disease) $[14,15]$.

On a local healthcare organizational level (e.g. hospital, clinic, long term care facility), technologies that have policies, procedures and processes embedded within electronic health records may not be consistent with emerging international and national knowledge about the disease. These technologies may in turn lead health professionals to engage in activities and workflows that are not consistent with the current knowledge of the disease. Healthcare organizations must modify their technologies to support disease management. This is a continuous, iterative process as information about the emerging disease is pushed out by international organizations and governments via websites and social media and healthcare organizations respond by modifying their HIT to reflect these changes in order to support health professional decision-making and work activities.

Healthcare organizations need to respond to new information by modifying the technologies that support their processes (e.g.
EHRs, DSS, telehealth systems, public health information systems) to be consistent with information being pushed out by international and local public health organizations. Healthcare organizations review emerging information from country and state public health organizations, develop and modify existing policies and procedures, identify methods of educating health professionals about the emerging disease, modify EHRs/DSS, deploy screening and develop HIT related strategies that facilitate communication among the members of the healthcare team. Such activities require that health informatics and HIT departments modify existing electronic tools (e.g. electronic screening, emergency room triage tools, clinical documentation, DSS, and laboratory and pharmacy information systems) to account for any additional/new laboratory tests, medications and other interventions that need to be employed with every new patient and their family and in keeping with best practices for the treatment of the emerging disease. It must be noted that healthcare organizations in such circumstances may not be able to modify the technology that supports patient care at the same pace or at all to integrate new information as it emerges. This inability to modify the technology or the lag time associated with technology change may lead health professionals to make decisions that are not consistent with current, patient-centric best practices involving the emerging disease. Inconsistencies between emerging patient centric treatments and the HIT could lead to the introduction of errors - technology-induced errors, where the technology is inconsistent with best practice [31].

\section{Reporting and Quality Im- provement Involving HIT}

Reporting medical incidents is one of the leading initiatives to enhance patient safety. Based on the successful safety improvements in aviation and nuclear plant industries, webbased voluntary medical incident reporting systems, i.e. e-reporting systems, are considered as an effective mechanism for learning from and preventing errors [51]. Such systems could offer a source of adverse event information, a reminder of hazards, and a means of monitoring potential problems as they recur. Ultimately, the systems would help researchers seek common solutions and translate reporting data into actionable knowledge.

Unfortunately, the current e-reporting systems are mostly used as a data repository due to the lack of structured data, uncertainty, ambiguity and incompleteness. Meanwhile, the systems also suffer from the issues of underreporting and low-quality reporting [52]. A significant percentage of submitted reports were incomplete or inaccurate, and thus cannot be thoroughly analyzed to understand the causes of medical errors [53-55].

The culture of blame and resistance to sharing has been identified as barriers to e-reporting at the organization level [56]. At the technology level, current e-reporting systems were not built on the basis of a consensus on the conceptual framework. Features that analyze medical errors collectively and facilitate learning have not been explored in current systems. One challenge of implementing such features is the inconsistency of data structure due to the difference in the conceptual framework, especially for home grown systems. In order to achieve the goal of preventing and reducing medical errors, e-reporting systems should be secure, easy to use and effective [54-57] - that is, confidential or anonymous, with excellent user acceptance, and used in a meaningful way. Being able to facilitate learning from past mistakes is critical to such systems to eventually decrease recurring incidents.

\section{Impact of Context}

Country context also has its impacts upon choice and use of HIT hardware and software during an outbreak of disease. There is a need to fully assess and understand the disease in the context of health system and HIT infrastructure of a country as suggested by Sittig and colleagues [58]. Developed and developing countries have differing needs. For example, the presence or absence of electricity, mobile phone coverage and the Internet influence what type of hardware is selected for clinical documentation. If 
there is limited electricity then some types of devices may be effective to use such as those that have long battery life (e.g. mobile phones and cameras to take pictures of paper documents versus laptop or desktop computers). Software (i.e. EHRs and DSS) need to be considered. Here, developed and developing countries employ these technologies in disease outbreak contexts. Many proprietary EHRs can be modified and DSS created to support clinician work as we have outlined earlier in this paper. In contrast many of the EHRs (including DSS) that are used in the developing world are open source. Morrison and colleagues [59] identify that many of the open source EHRs lack the essential characteristics of a HIT that can fully support management of new/emerging diseases such as the ability to quickly embed treatment and research protocols, create reminders and measure response to treatment of disease. Buhler et. al. [60] note healthcare workers, who are in the midst of caring for patients with such diseases, are more concerned about patients who are suffering from illness, having sufficient people to care for patients and their families, and having access to protective clothing to prevent further spread of the disease. They note that laptops, mobile phones and cameras (used to photograph the paper chart) are a concern for healthcare professionals. The belief is that HIT may become contaminated and that current approaches to decontaminating the technology may damage the HIT itself [59]. Some of these challenges are dependent on country context.

\section{Summary and Conclusions}

In summary, new/emerging diseases present a number of opportunities for HIT innovation. These may include the development of software applications and devices for tracking, methods of delivering tailored, patient centric information to large populations of individuals and for developing new EHR, public health system and decision support system functionality. The pressures placed on HIT by new/emerging diseases can lead to innovation and improvements in patient centric care and patient safety. An educated population is better able to prevent disease and understand how to seek medical attention when needed.

Here, the Internet, search engines and social media play a significant and important role for the public, health professionals and healthcare organizations in preventing disease spread. Tools such as WHO and CDC websites disseminate important information to all who have access to the WWW and search engines using fixed (e.g. laptop) and mobile devices (e.g. Mobile phones). Software applications in conjunction with devices can be used to push information (e.g. Twitter ${ }^{\circledR}$, Youtube $\left.{ }^{\circledR}\right)$, support self-monitoring for disease symptoms, and be used to call for help.

Alternatively, many challenges still remain from a contextual, human factors, organizational, inter-organizational and design perspective. HIT researchers must not only develop HIT that supports surveillance, screen for, diagnosis and management of emerging/new disease, but they also need to develop methods to rapidly create new technologies and/or optimize existing ones to reflect the changing information and knowledge we have about a new and emerging disease so no new errors are created when there are inconsistencies between the emerging science surrounding a disease and the technology used to support patient-centred care. Achieving patient-centered care will require greater consideration of a range of issues particularly in the age of emerging diseases and epidemics.

\section{References}

1. Ryan R, Santesso N, Lowe D, Hill S, Grimshaw J, Prictor M, et al. Interventions to improve safe and effective medicines use by consumers: an overview of systematic reviews. Cochrane Database Syst Rev 2014;4.

2. McCarthy DM, Davis TC, King JP, Mullen RJ, Bailey SC, Serper M, et al. Take-Wait-Stop: a patient-centered strategy for writing PRN medication instructions. J Health Commun 2013;18(Suppl 1):40-8.

3. Huber J, Ihrig A, Winkler E, Winkler E, Brechtel A, Friederich HC, Herzog W, et al. Interdisciplinary counseling service for renal malignancies: A patient-centered approach to raise guideline adherence. Urol Oncol 2014 Oct 30.

4. McMullen CK, Safford MM, Bosworth HB,
Phansalkar S, Leong A, Fagan MB, et al. Patient-centered priorities for improving medication management and adherence. Patient Educ Couns 2014;98(1):102-10

5. Reed SC, Partridge AH, Nekhlyudov L. Shared Medical Appointments in Cancer Survivorship Care: A Review of the Literature. J Oncol Pract 2014 Nov 25

6. Gulliford M, Smriti N, Myfanway M. What is 'continuity of care'? J Health Serv Res Policy 2006 Oct;11(4):248-50.

7. Hernandez LM. Health literacy, eHealth and communication: Putting the consumer first workshop summary. Institute of Medicine: Washington, D. C; 2009.

8. Simon AC, Gude WT, Holleman F, Hoekstra JB, Peek N. Diabetes patients' experiences with the implementation of insulin therapy and their perceptions of computer-assisted self-management systems for insulin therapy. J Med Internet Res 2014;16(10):e235.

9. Magnuson JA, Fu PC. Public Health Informatics and Information Systems. London: Springer; 2014.

10. Borycki EM. Technology-induced errors: where do they come from and what can we do about them? Stud Health Tech Inform 2013,194;20-6.

11. Kuziemsky CE, Borycki EM, Purkis ME, Black F, Boyle M, Cloutier-Fisher D, et al. An interdisciplinary team communication framework and its application to healthcare'e-teams' systems design. BMC Med Inform Decis Mak 2009 Sep 15;9:43.

12. Pendergrast M. Inside the outbreaks. Boston: Mariner Books; 2011.

13. Frisch LF, Borycki EM, Capron A, Mawudeku A, John RS. Public Health Informatics in Canada. In; Public Health Informatics and Information Systems. London: Springer; 2014. P. 603-18.

14. Zhang J, Sun J, Yang Y, Chen X, Meng L, Lian P. Web-based electronic patient records for collaborative medical applications. Comput Med Imaging Graph 2005 Mar-Apr;29(2-3):115-24.

15. Valaitis RK, Akhtar-Danesh N, Kealey CM, Brunetti GM, Thomas H. A severe acute respiratory syndrome extranet: Supporting local communication and information dissemination. BMC BMC Med Inform Decis Mak 2005;5(1);17.

16. Centres for Disease Control and Prevention. Case definition for Ebola virus disease (EVD). 2014. http://www.cdc.gov/vhf/ebola/hcp/case-definition. html (accessed on December 15, 2014.)

17. World Health Organization. Ebola virus disease. http://www.who.int/mediacentre/factsheets/fs103/ en (accessed on December 15, 2014)

18. BBC News. Ebola: Mapping the outbreak. December 16, 2014. http://www.bbc.com/news/ world-africa-28755033?print=true. (accessed on December 15, 2014.)

19. Sittig DE, Singh H. A new socio-technical model for studying health information technology in complex adaptive healthcare systems. Qual Saf Health Care 2010;19(3):68-74.

20. Lee K, Hoti K, Hughes DJ, Emmerton L. Dr Google and the Consumer: A Qualitative Study Exploring the Navigational Needs and Online Health Information-Seeking Behaviors of Consumers With Chronic Health Conditions J Med Internet Res 2014;16(12):e262. 
21. Zulman MD, Kirch M, Zheng K, An CL. Trust in the Internet as a Health Resource Among Older Adults: Analysis of Data from a Nationally Representative Survey. J Med Internet Res 2011;13(1):e19.

22. Wagner MM, Tsui FC, Espino JU, Dato VM, Sittig DF, Caruana RA, et al. The emerging science of very early detection of disease outbreaks. J Public Health Manag Pract 2001 Nov;7(6):51-9.

23. K. Purcell, L. Rainie. Americans Feel Better Informed Thanks to the Internet. Pew Internet Research Project. 2014. Retrieved from http://www. pewinternet.org/2014/12/08/better-informed/

24. Boyer C, Gaudinat A, Baujard V, Geissbuhler A. Health on the Net Foundation: assessing the quality of health web pages all over the world, in Medinfo 2007: Proceedings of the 12th World Congress on Health (Medical) Informatics; Building Sustainable Health Systems, 2007. p. 1017.

25. Peterson G, Aslani P, Williams AK, How do Consumers Search for and Appraise Information on Medicines on the Internet? A Qualitative Study Using Focus Groups. J Med Internet Res 2003;5(4):e33.

26. ITU, The World in 2010: ICT facts and figures, available from: http://www.itu.int/en/ITU-D/ Statistics/Documents/facts/ICTFactsFigures2010. pdf\#search=world $\% 20$ mobile $\% 20$ uptake, Accessed 30/12/2014

27. Prescott R. M-health: 2.8 million patients remotely monitored worldwide in 2012, available from http://www.rcrwireless.com/20130116/wireless/2-8-m-patients-remotely-monitored-worldwide-2012, accessed 30/12/2014.

28. Sahota D. Africa gets smart: continent prepares for device revolution January 9, 2014, available at: http://www.telecoms.com/209421/africa-gets-smart-continent-prepares-for-device-revolution/, accessed December 42014.

29. Whitney L. LG to donate 2,000 smartphones to UN's Ebola effort. 24 October 2014 http://www. cnet.com/au/news/lg-to-donate-2000-smartphones-to-aid-uns-ebola-effort/

30. Upadhyay D, Sittig DF, Singh H. Ebola US patient zero: lessons on misdiagnosis and effective use of electronic health records [ePub ahead of print]. Diagnosis 2014.

31. Borycki EM, Kushniruk AW. Where do technology-induced errors come from? Towards a model for conceptualizing and diagnosing errors caused by technology. Human, Social, and Organizational Aspects of Health Information Systems. Hershey, Pennsylvania: Idea Group;2008.

32. Kushniruk AW, Triola MM, Borycki EM, Stein B, Kannry JL. Technology induced error and usability: The relationship between usability problems and prescription errors when using a handheld application. Int J Med Inform 2005;74(7):519-26.

33. Varga D. Examing the U.S. public health response to the Ebola outbreak. Testimony, October 16, 2014, before the U.S. House Energy and Com- merce Committe and Subcommittee on Oversight and Investigations; 2014.

34. Musen MA, ShakarY, Shortliffe EH. Clinical Decision-Support Systems In: Shortliffe EH, Cimino JJ, editors. Biomedical informatics: computer applications in health care and biomedicine. 3rd ed. New York: Springer; 2006. p. 698-736.

35. Slagle JM, Gordon JS, Harris CE, Davison CL, Culpepper DK, Scott P, et al. MyMediHealth - designing a next generation system for child-centered medication management J Biomed Inform 2010 Oct;43(5 Suppl):S27-31.

36. Chute CG, Beck SA, Fisk TB, Mohr DN. The Enterprise Data Trust at Mayo Clinic: a semantically integrated warehouse of biomedical data. J Am Med Inform Assoc. 2010 Mar-Apr;17(2):131-5.

37. Bell LM, Grundmeier R, Localio R, Zorc J, Fiks AG, Zhang $X$, et al. Electronic health record-based decision support to improve asthma care: a cluster-randomized trial. Pediatrics 2010 Apr;125(4):e770-7.

38. Charles D, Gabriel M, Furukawa M. Adoption of electronic health record systems among U.S. non-federal acute care hospitals: 2008-2013. ONC data brief no. 16. Washington, DC: Office of the National Coordinator for Health Information Technology; May 2014.

39. Mandl KD. Ebola in the United States: EHRs as a Public Health Tool at the Point of Care. JAMA 2014 Dec 17;312(23):2499-500.

40. Wright A, Sittig DF. SANDS: A service-oriented architecture for clinical decision support in a national health information network; J Biomed Inform 2008;41:962-81.

41. Lurio J, Morrison FP, Pichardo M, Berg R, Buck MD, Wu W, et al. Using electronic health record alerts to provide public health situational awareness to clinicians. J Am Med Inform Assoc 2010;17(2);217-9.

42. Collins SA, Bakken S, Vawdrey DK, Coiera E, Currie L. Model development for EHR interdisciplinary information exchange of ICU common goals. Int J Med Inform 2011;80(8);e141-e149

43. Samsung fight ebola with smartphone donation. http://www.itwebafrica.com/mobile/435-sierra-leone/233667-samsung-fights-ebola-with-smartphone-donation

44. Tomines A, Readhead H, Readhead A, Teutsch S. Applications of Electronic Health Information in Public Health: Uses, Opportunities and Barriers. eGEMs (Generating Evidence \& Methods to improve patient outcomes) 2013;1( 2), article 5.

45. Birkhead GS, Klompas M, Shah NR. Uses of electronic health records for public health surveillance to advance public health. Annu Rev Public Health 2015;36(15),1-15,15.

46. Kuehn BM. Report: States May Be Unprepared to Manage Infectious Disease Threats. JAMA 2014;311(4):348.

47. Klompas M, McVetta J, Lazarus R, Eggleston E, Haney G, Kruskal BA, et al. Integrating clinical practice and public health surveillance using electronic medical record systems. Am J Public Health 2012;102(S3);325-32.

48. Kukafka R, Ancker JS, Chan C, Chelico J, Khan $\mathrm{S}$, Mortoti S, et al. Redesigning electronic health record systems to support public health. J Biomed Inf 2007;40(4):398-409.

49. Hanauer DA, Zheng K, Mei Q, Choi SW. Full-text search in electronic health records: Challenges and opportunities. Internet Policies 2009;7:745-8.

50. Kushniruk AW, Patel VL. Cognitive and usability engineering methods for the evaluation of clinical information systems. J Biomed Inform 2004;37(1);56-76.

51. Borycki EM, Kushniruk A, Keay E, Nicoll J, Anderson J, Anderson M. Toward an integrated simulation approach for predicting and preventing technology-induced errors in healthcare: implications for healthcare decision-makers. Healthc Q 2009;12 Spec No Patient:90-6.

52. Thomas EJ, Petersen LA. Measuring errors and adverse events in health care. J Gen Intern Med 2003;18(1);61-7.

53. Cohen MR. Why error reporting systems should be voluntary. BMJ 2000;320(7237):728-9.

54. Gong Y. Data consistency in a voluntary medical incident reporting system. J Med Syst 2011;35(4):609-15.

55. Leape LL. Reporting of medical errors: time for a reality check. Qual Health Care 2000 Sep;9(3):144-5.

56. Tighe CM, Woloshynowych M, Brown R, Wears B, Vincent $C$. Incident reporting in one UK accident and emergency department. Accident and Emergency Nursing 2006;14(1):27-37.

57. Barach P, Small SD. Reporting and preventing medical mishaps: lessons from non-medical near miss reporting systems. BMJ 2000;320(7237):759-63.

58. Sittig DF, Kahol K, Singh H. Sociotechnical evaluation of the safety and effectiveness of pointof-care mobile computing devices: A case study conducted in India. Stud Health Technol Inform 2013;192:515-9.

59. Morrison C, Iosif A, Danka M. Report on existing open-source electronic medical records. Technical Report. Cambridge: United Kingdom; 2010.

60. Buhler S, Roddy P, Nolte E, Borchert M. Clinical documentation and data transfer from Ebola and Marburg virus disease wards in outbreak settings: Health care workers' experiences and preferences. Viruses 2014;6:927-37.

\section{Correspondence to:}

Elizabeth Borycki

Health Information Science

University of Victoria

PO Box 1700 STN CSC

Victoria BC V8W 2Y2, Canada

E-Mail: emb@uvic.ca 